\title{
Evaluación de una intervención logopédica en pacientes con la enfermedad de Alzheimer en tratamiento colinérgico: Un estudio piloto
}

\author{
Miguel López-Zamora, Miriam Cánovas-Cano y Lourdes Aranda \\ Universidad de Málaga, Málaga, España
}

\begin{abstract}
Evaluation of a speech therapy in patients with Alzheimer's disease under cholinergic treatment: A pilot study. The intervention on Alzheimer's disease (AD) has shown that pharmacological treatments with acetylcholinesterase inhibitors are highly beneficial, but there is not much evidence of the efficacy of other treatments, as it is difficult to find non-medicated patients. In the present pilot study, the efficacy of a logopedic intervention was verified in patients with AD in the mild phase, with and without pharmacological treatment. The results show that the logopedic intervention slows the deterioration of the disease. The combination of logopedic intervention and pharmacological therapy is confirmed as the therapeutic option that most benefits the patient, whilst patients who only received one of the treatments showed a more pronounced cognitive deterioration. The conclusions indicate that the pharmacological approach is necessary but insufficient, and that a logopedic intervention may be an adequate and effective complement in $\mathrm{AD}$.
\end{abstract}

Keywords: Alzheimer's disease; speech therapy; acetylcholinesterase inhibitors.

Resumen: Al intervenir en pacientes con la enfermedad de Alzheimer (EA) se ha comprobado que los tratamientos farmacológicos con inhibidores de acetilcolinesterasa resultan altamente beneficiosos, pero existen pocas evidencias sobre la efectividad de otros tratamientos debido a que es difícil encontrar pacientes que no estén medicados. En el presente estudio piloto se comprueba la efectividad de una intervención logopédica en enfermos con EA en fase leve, con y sin tratamiento farmacológico. Los resultados muestran que la intervención logopédica frena el deterioro de la enfermedad. La combinación de intervención logopédica y terapia farmacológica es la opción terapéutica que más beneficios reporta al paciente, mientras que los enfermos que sólo recibieron uno de los tratamientos mostraron un deterioro cognitivo más pronunciado. Las conclusiones indican que la aproximación farmacológica es necesaria pero insuficiente, y que una intervención logopédica puede ser un complemento adecuado y eficaz en la EA.

Palabras clave: Enfermedad de Alzheimer; Intervención logopédica; inhibidores de acetilcolinesterasa.

\section{Introducción}

El envejecimiento de la población mundial es un problema de primer nivel que ha ido aumentando progresi-

Recibido: 24 de octubre 2018; aceptado: 22 de febrero 2019 Correspondencia: Miguel López Zamora, Facultad de Psicología y Logopedia. Málaga, Campus de Teatinos s/n, 29010 Málaga, España. Correo-e: miguel.lopez@uma.es.

Agradecimientos: Queremos agradecer la inestimable colaboración a la Asociación de Familiares de enfermos de Alzheimer y otras demencias de Málaga (AFA), y a la logopeda y terapeuta ocupacional Noelia Guzmán Usero, que nos asesoró durante toda la intervención. Este trabajo ha sido financiado por el Plan Propio de Investigación y Transferencia de la Universidad de Málaga. vamente su importancia en la agenda de todos los gobiernos del primer mundo y, en concreto, para los países europeos. En el caso de España, el incremento de la esperanza de vida y el descenso de la natalidad ha provocado que en 2017 el $25.3 \%$ de la población supere los 60 años, porcentaje que alcanzará el $41.9 \%$ en el año 2050 (UN, 2017). Este problema demográfico plantea numerosos desafíos económicos y sociales de gran calado, siendo uno de los que mas preocupan el aumento de personas con enfermedades crónicas invalidantes, como la enfermedad de Alzheimer (EA). La EA es el tipo más frecuente de demencia, caracterizado por una alteración neural irreversible que produce alteraciones en la memoria, en la personalidad y en el razonamiento, y que en 
Europa presenta una prevalencia 4.66\% (Niu, Álvarez-Álvarez, Guillén-Grima, y Aguinaga-Ontoso, 2018). La EA se manifiesta como una disminución de la capacidad funcional y cognitiva, donde el paciente va perdiendo todas sus capacidades verbales y psicomotoras, junto con la presencia de síntomas neuropsiquiátricos tan invalidantes que el paciente se vuelve totalmente dependiente (Burns y Iliffe, 2009). De inicio insidioso y lento, suele ser detectada de manera tardía, y en la actualidad no tiene vacuna ni cura posible. El carácter irreversible de la EA obliga a que, una vez sea detectada, se intervenga lo más pronto posible, con el objetivo de retrasar el deterioro cognitivo y conservar la calidad de vida del paciente el máximo tiempo posible. En la actualidad existen dos tipos de aproximaciones terapéuticas a la EA, los tratamientos farmacológicos y las intervenciones neurocognitivas.

Las terapias farmacológicas más eficaces para detener el progresivo avance de la EA son las que se basan en el suministro de inhibidores de la acetilcolinesterasa (ICE), donepecilo, galantamina y rivastigmina, cuya función principal es hidrolizar la acetilcolina (Sun, Jin, y Ling, 2012; Kumar, Singh, y Ekavali, 2015; Boccardi et al., 2016). La acetilcolina es la sustancia química que actúa en la transmisión de los impulsos nerviosos. Sintetizada por el sistema colinérgico del Sistema Nervioso Central, media la actividad sináptica en el cerebro y regula la plasticidad neuronal, dos de las funciones más afectadas por la EA (Llanero, Ruiz, Medrano, y Fernández, 2011; Zamarrón, Tárraga, y Fernández-Ballesteros, 2008; Peña-Casanova, 1999). Los tratamientos con ICE compensan la disfunción que se da en la vía colinérgica y mantienen niveles de acetilcolina normalizados (López-Locanto, 2015, para un resumen de los fármacos utilizados para tratar la EA). Existe otro medicamento, la memantina, que actúa sobre el sistema glutamatérgico y posee funciones neuroprotectoras sobre las áreas de la memoria y del aprendizaje, y que suele ser prescrito junto con los ICE en los tratamientos farmacológicos para pacientes que se encuentran en la fase moderada o grave. Estos dos medicamentos son los más utilizados para tratar la EA, pero sus resultados distan mucho de ser homogéneos.

En los últimos años han surgido estudios discordantes acerca de la preponderancia que se le da, en términos absolutos, a las investigaciones farmacológicas. Sin restarle importancia a su enorme éxito global, dependiendo de la edad de aparición de la EA (Gallucci, Spagnolo, Aricó, y Grossi, 2016), el nivel de estudios (Valenzuela, 2008), la respuesta inicial al tratamiento (Boccardi et al., 2016) o el género (Mielke, Vemuri, y Rocca, 2014), la respuesta a la intervención farmacológica puede variar de una investigación a otra. Independientemente de las diferencias individuales de los pacientes, también se ha constatado que los ICE no están exentos de problemas, pudiendo producir alteraciones cognitivas y motoras (Hernández-Arroyo y Díaz-Madero, 2016) o perder su efecto dependiendo del nivel de deterioro cognitivo del paciente, hasta tal punto que Livingston et al., (2017) han demostrado que en fase moderada y avanzada ya no son eficaces.

La intervención no farmacológica es la segunda aproximación terapéutica a la EA más extendida, y se define como cualquier intervención no química, focalizada, replicable, y con capacidad potencial de lograr algún beneficio relevante en el paciente (Olazarán et al., 2010). Las intervenciones más utilizadas son las que se centran en la recuperación de las funciones cognitivas (Peña-Casanova, 1999), como la Terapia de Estimulación Cognitiva de Mantenimiento (MCST; Aguirre et al., 2010) o el Programa de Psicoestimulación Integral (PPI) de Tárraga (2001). Sin embargo, existen otros tipos de intervenciones de carácter heterogéneo, cuyo rango de éxito muy variado, y que destacan por presentar una metodología poco sistemática. Las más conocidas son la musicoterapia (García-Casares, Moreno-Leiva, y García-Arnés, 2017), las intervenciones asistidas con animales (Muñoz-Lasa et al., 2015), las focalizadas en reducir la depresión (García-Alberca, 2015) o las hidroterapias, como la basada en el surf (Real, Robles, y Ponce, 2017). Esta falta de sistematicidad implica una replicabilidad complicada, de manera que es muy difícil comprobar su eficiencia terapéutica. Un ejemplo de esta problemática es la que ha surgido en la investigación de la relación entre la EA y el ejercicio físico. Desde principios de este siglo se han generalizado intervenciones que afirman que una rutina de estimulación física produce un impacto beneficioso en los procesos cognitivos de los enfermos de la EA (Burge, Kuhne, Berchtold, Maupetit, y von Gunten, 2011). Sin embargo, investigaciones recientes sobre dicha cuestión (Frederiksen, Gjerum, Waldemar, y Hasselbalch, 2018; Van der Kleij et al., 2018) demuestran que el ejercicio no produce ninguna mejoría directa en ningún marcador neuronal de los pacientes con la EA. El anterior es sólo un ejemplo de cómo, durante muchos años, se ha generalizado la errónea idea de que cualquier terapia que se le aplique al paciente tendrá un efecto beneficioso para el enfermo, sin que importe comprobar la validez metodológica o terapéutica de la intervención. Esto tiene importantes consecuencias económicas, terapéuticas y éticas en todos los niveles sanitarios, puesto que aplicar un tratamiento erróneo no sólo tiene un importante coste, sino que además impide que el paciente reciba uno más adecuado a 
sus circunstancias. Por estas razones es importante comprobar de manera sistemática que las metodologías aplicadas hoy en día son capaces de incidir de manera exitosa en los pacientes.

Las intervenciones no farmacológicas más extendidas en la EA son las cognitivas, pero tampoco están exentas de críticas. Zamarrón et al. (2008) demostraron que muchas terapias cognitivas producen mejorías marginales en los enfermos, y que su principal utilidad vendría a ser la de un método paliativo para preservar la capacidad de aprendizaje. Esta falta de eficiencia se ha mostrado incluso en intervenciones con pacientes sanos. Novoa, Juárez, y Nebot (2008) llevaron a cabo una revisión de 25 estudios con ancianos con deterioro cognitivo, acerca de la efectividad de la intervención cognitiva sobre la memoria. Aunque encontraron que determinadas intervenciones parecían eficientes, su revisión mostró que 14 de los estudios revisados no cumplían unos mínimos criterios de calidad metodológica. Por estas razones debemos ser prudentes con las intervenciones se aplican. Ruiz-Sánchez de León (2012) denomina «pseudoprogramas» a aquellas intervenciones que, afirmando trabajar la estimulación cognitiva, únicamente consisten en meras repeticiones de ejercicios sin metodología definida. Para este autor, la administración individualizada o en grupos pequeños, y la selección de tareas con una base teórica es condición imprescindible para hacer una intervención exitosa, lo que no es frecuente. Precisamente el formato de la intervención individual o grupal en la estimulación cognitiva en la EA ha sido estudiado recientemente, encontrándose que, si bien los enfermos siempre evolucionan positivamente en una intervención cognitiva, ésta es mas exitosa cuando es aplicada individualmente (Fernández-Calvo, Contador, Serna, Menendez de Lucena, y Ramos, 2010). Por último, además de los problemas mencionados se suma un factor complementario, y es que la mayoría de los enfermos con la EA son medicados inmediatamente tras el diagnóstico inicial, por lo que es complicado conocer la magnitud del beneficio individual de las intervenciones cognitivas independientemente de su tratamiento farmacológico (García-Alberca, 2015).

Cabe mencionar que, dentro de las estimulaciones cognitivas, el lenguaje suele ser tratado como un factor más a cuantificar e intervenir. Es llamativo que las intervenciones desde un punto de vista logopédico sean tan escasas dado el amplio rango de síntomas lingüísticos posibles. Un paciente con la EA puede mostrar problemas en nombrado, comprensión lectora y auditiva, habla fluida pero vacía, parafasia semántica, y alteraciones sintácticas y morfológicas. Además, pueden aparecer problemas en los órganos fonadores, trastornos del ritmo verbal como modificaciones de la intensidad, retraso en el habla retardada, verbigeración, logorrea, logoclonía, mutismo, tartamudez o ecolalia (Sun et al., 2012; Aranda, Gordillo-Villatoro, Enamorado, y López-Zamora, 2017). Intervenir el lenguaje de manera paralela y especializada al resto de problemas cognitivos presenta la ventaja de que los pacientes tendrán una mayor protección de la función comunicativa que les permita relacionarse con su contexto social y familiar, evitando su aislamiento $\mathrm{y}$, como consecuencia, ralentizando la disminución de su calidad de vida (Cullell, Bruna, y Puyuelo, 2006; Klimova, Maresova, Valis, Hort, y Kuca, 2015). Por esta razón es esencial realizar no sólo una aproximación multidisciplinar donde estén presentes médicos especialistas, psicólogos, terapeutas ocupacionales, fisioterapeutas o la propia familia, sino también los logopedas, que tienen un papel fundamental en el trabajo con estos pacientes, ayudándoles de esta forma a preservar sus funciones comunicativas.

Por todo lo anteriormente expuesto, el presente trabajo se planteó con el propósito de estudiar la efectividad de un programa de intervención logopédica en pacientes diagnosticados con la EA en fase leve. Esta investigación se articuló en torno a dos objetivos. En primer lugar, estudiar la efectividad de la intervención logopédica en enfermos de la EA. En segundo lugar, comprobar si la intervención logopédica preservó algún área cognitiva y si hubo alguna que mostrase alguna mejoría. La hipótesis de este trabajo se centró en las capacidades lingüísticas de los pacientes. Debido la intervención incidió directamente en las habilidades del lenguaje del enfermo, se hipotetizó que, como mínimo, todas las áreas lingüísticas aparecerían preservadas al final de la intervención y que si se encontraba alguna mejoraría, sería en una de estas áreas. El grupo muestral fue dividido en cuatro grupos según las condiciones experimentales: Un primer grupo control, un segundo grupo con intervención logopédica, un tercero con tratamiento farmacológico y, por último, un grupo combinado con intervención logopédica y tratamiento farmacológico. Para evaluar la efectividad de la intervención logopédica se aplicó un diseño test-postest a partir de los resultados en la Escala Pro-Neuro de Evaluación Cognitiva-Lingüística (Adrián, Hermoso, Buiza, Rodríguez-Parra, y González, 2008).

\section{Método}

\section{Participantes}

En este estudio participaron 16 pacientes, 9 mujeres y 7 hombres $(M=75.9$ años; $D T=6.7)$, miembros de la Asociación de Familiares de enfermos de Alzheimer y 
otras demencias de Málaga (AFA), y derivados por los servicios de neurología de sus centros médicos con los requerimientos de ser enfermos de la EA en fase leve y no sufrir ninguna otra patología susceptible de alterar los resultados de esta investigación. Se obtuvo un consentimiento firmado de todos los participantes y de sus familiares cuando el sujeto tuvo problemas para entender las implicaciones éticas del experimento.

Para la selección de la muestra se llevaron a cabo dos entrevistas semiestructuradas, con los familiares de los pacientes y con los cuidadores de la asociación, con los objetivos de contrastar el diagnóstico del Servicio Andaluz de Salud (SAS) con los informes de los cuidadores de la asociación, obtener una anamnesis completa por parte de los familiares y, por último, de estudiar la dinámica del paciente con ambos grupos de personas. Se llevaron a cabo 25 entrevistas para la selección inicial de los sujetos, que sirvieron para descartar a siete pacientes por sufrir demencias vasculares, semánticas, frontotemporales o de cuerpos de Lewy. Durante el tiempo del proceso de las entrevistas uno de los pacientes falleció y otro fue trasladado de centro, por lo que fueron excluidos de la muestra, lo que fijó la muestra final en 16 sujetos.

El grupo muestral se dividió en cuatro grupos de cuatro pacientes según el tratamiento aplicado: Grupo control, grupo con intervención logopédica, grupo con tratamiento farmacológico, y grupo combinado con intervención logopédica y tratamiento farmacológico. Los pacientes farmacológicos seleccionados tuvieron el requisito adicional de estar recibiendo un tratamiento estable con ICE de, como mínimo, 6 meses antes de esta investigación. La inclusión en la condición de intervención logopédica fue aleatorizada.

\section{Procedimiento}

Las pruebas fueron aplicadas por la logopeda de la Asociación AFA, en conjunción con una alumna en prácticas del último curso del grado de Logopedia, y cada intervención duró entre once y doce semanas, aunque debido a que en la asociación AFA no tenían inicialmente el número mínimo de sujetos para completar cada condición, hubo que esperar a que el SAS derivase a candidatos susceptibles de ser entrevistados y, después, de ser incluidos en el grupo correspondiente. Por esta razón, la pasación comenzó en noviembre de 2015 y las intervenciones se fueron aplicando en paralelo hasta que se completaron los grupos muestrales en mayo de 2016. La intervención de cada sujetó comenzó con la selección inicial de la muestra, las entrevistas, la familiarización de los pacientes a la alumna en prácticas, y la evaluación pretest. En las siguientes nueve semanas se llevó a cabo la intervención logopédica, mientras que en las dos últimas semanas se llevó a cabo la evaluación postest.

Se planificaron 18 sesiones de intervención logopédica de 30-45 minutos de duración durante dos meses, a razón de dos cada semana. Las intervenciones se pasaron individualmente junto con las dos terapeutas, en una sala aislada de la asociación que fuese familiar para el paciente. Todas las sesiones se programaron en el mismo horario, con el objetivo de establecer rutinas fijas, y aunque se trabajaron seis áreas previstas en cada sesión, el uso de los materiales y la aplicación de las actividades dependió de factores como la actitud colaboradora o del estado de las capacidades cognitivas y/o emocionales del paciente. Excepto cuando se trabajó el área de la memoria, se procuró presentar materiales novedosos en cada sesión para evitar que el paciente acertase debido que reconociese el estímulo de la tarea.

\section{Instrumentos}

Mini-Mental State Examination (MMSE, Folstein, Folstein, McHugh, y Fanjiang, 1975; versión española de Lobo et al. (1999). Es una prueba que dura entre 5 y 10 minutos, se utilizó únicamente para detectar el deterioro cognitivo de los pacientes antes de la intervención logopédica. Consta de 30 preguntas que evalúan orientación espacio temporal, atención, concentración y memoria, capacidad de abstracción y cálculo, lenguaje y percepción visoespacial, y capacidad para seguir instrucciones básicas.

Escala Pro-Neuro de Evaluación Cognitiva-Lingüistica (Adrián et al, 2008). Fue utilizada para evaluar en las fases pretest y postest, y consta de pruebas breves que miden de forma cuantitativa la orientación temporal-espacial, atención y memoria, cálculo mental, comprensión verbal, escritura, denominación, razonamiento, fluidez verbal, praxias y gnosia visual, ofreciendo una puntuación total que representa la situación cognitiva del paciente. El alfa de Cronbach para este estudio fue de .73 .

\section{Intervención}

La intervención trabajó seis áreas cognitivo-logopédicas:

a) Orientación temporal, espacial y personal. Consistió en una entrevista breve en la que se hacían preguntas relacionadas con las áreas trabajadas. En la orientación temporal se preguntaba sobre eventos cronológicos relacionados con su vida. En orientación espacial acerca de lugares donde el sujeto había vivido o conocido. En 
orientación personal sobre su vida personal, parentesco o eventos vividos o conocidos. Si el paciente tenía dificultades para contestar, se le apoyaba con frases tipo cloze (v.g.: «Si hoy es viernes, mañana es...»). Las preguntas con más dificultades se anotaban y se trabajaban en la segunda sesión semanal para reforzarlas, puesto que, en la fase leve de las demencias las áreas más afectadas son la orientación y la cronología (Tárraga y Boada, 1999). Si el paciente estaba colaborador se extendía la duración de la sesión para promover, paralelamente, el lenguaje espontáneo, y la memoria verbal y episódica.

b) Lenguaje. En la fase leve de la EA el lenguaje suele ser fluido, pero hay que tener en consideración la frecuente presencia de parafasias, circunloquios, pausas para poder encontrar la palabra adecuada y anomia. El lenguaje automático se trabajó mediante la evocación de números, días de la semana, meses del año, trozos de refranes y canciones. El lenguaje espontáneo se trabajó mediante la descripción de láminas e imágenes, ofreciéndoles ayuda si no había respuesta a los 30 segundos. La comprensión y el razonamiento verbal fueron una de las áreas con más actividades planeadas por ser las que más rápidamente se deterioran en la EA (Klimova et al., 2015). Se diseñaron 8 actividades con el objeto de estimular la semántica y el acceso al léxico, y en cada sesión se trabajaban dos, de manera que fueran rotando. Las actividades fueron las siguientes: 1) Agrupación de palabras e imágenes distribuidas por categorías y campos semánticos $\mathrm{y}$, ejercicios de emparejamiento de iguales por categorías a nivel visual y/o escrito. Se preguntaba qué rasgos eran los que unían los elementos presentados para estimular su razonamiento verbal; 2) Actividades donde se les presentaba una lámina de 4 imágenes, en la que había que seleccionar aquella que no pertenecía a la misma categoría. Para aumentar la dificultad se realizaba con sinónimos de forma escrita o con categorías más específicas; 3) Descomponer verbalmente o de manera escrita un objeto, evocando al menos tres elementos que formasen parte de éste. Si el paciente tenía dificultades se les presentaba el apoyo visual del objeto real o imagen, señalándole los componentes y ofreciéndole claves fonológicas; 4) Frases tipo «cloze» con y sin alternativas de respuesta; 5) Tareas de denominación por confrontación visual de imágenes cotidianas en tarjetas, con posteriores preguntas acerca de su utilidad, composición o características; 6) Denominación a través de definiciones; 7) Emparejamiento palabra y oración hablada-dibujo; 8) Tareas de corrección de frases escritas con elementos intrusos que no guardaban relación con el resto de los elementos. El paciente debía detectar el fallo y escribir una palabra correcta en su lugar. c) Fluidez verbal. En estos ejercicios, el paciente debía evocar palabras de una categoría concreta durante un tiempo determinado. Se utilizaron categorías generales como colores, frutas o animales y otras más específicas como épocas del año o tipos de vehículos. Durante el primer mes de intervención no se marcó un tiempo máximo para familiarizar al paciente con la tarea, pero en el último mes se puso un máximo de un minuto por categoría para cuantificar su progreso. En el caso de la evocación se utilizaron ejercicios de complejidad creciente donde el paciente tenía que realizar un razonamiento lógico, como «dígame una fruta que sea más blanda y más pequeña que una sandía».

d) Lectura y escritura. Se utilizaron lectura de oraciones y textos, copia de frases, escritura de frases al dictado y realización de frases a partir de una palabra determinada. Después de cada actividad se les hacía preguntas cerradas (sí/no) o en las que tenían que razonar acerca de alguna escena de la historia, trabajando la comprensión y la expresión verbal. Estos ejercicios duraban diez minutos y los textos fueron seleccionados específicamente para cada paciente de acuerdo a sus capacidades y preferencias.

e) Funciones ejecutivas y praxias. En la función ejecutiva se usaron actividades de búsqueda de semejanzas y diferencias de elementos presentados de forma oral, ordenación de palabras para crear frases con sentido mediante recortables, ordenación de secuencias narrativas mediante imágenes y realización de órdenes sencillas de complejidad creciente. Estas actividades además sirvieron para trabajar atención, memoria y praxias. Se trabajaron tres tipos de praxias: ideomotora (ejecutar un gesto simple de orden o de imitación), ideatoria (hacer secuencias gestuales y/o utilizar objetos para su realización) y constructiva (reproducir un modelo o dibujo, a la orden o a la copia).

f) Memoria verbal, atención y memoria. Para la memoria inmediata se usaron las mismas láminas trabajadas en lenguaje espontáneo. Se enseñaban, se retiraban y posteriormente se debía recordar qué elementos aparecían en ella. En otro ejercicio se utilizó un ábaco con bolas de colores para trabajar estas áreas junto con las funciones ejecutivas. La tarea consistió en escuchar instrucciones sobre cálculos y posiciones de las bolas, para después ejecutarlas y recordarlas, lo que favoreció la comprensión de órdenes, las praxias y la memoria de dígitos. La memoria visoespacial se trabajó de la misma manera que la memoria inmediata, mediante la descripción y retirada de láminas, pero en este caso se le preguntaba por la localización de elementos descritos en el espacio. Por último, la memoria auditivo-verbal se trabajó mediante la repetición de palabras, frases y dígitos. 


\section{Análisis estadístico}

El análisis de datos se llevó a cambio mediante el software estadístico SPSS 23. En primer lugar, se llevó a cabo un análisis de varianza (ANOVA) de las puntuaciones totales discretas del Pro-Neuro para estudiar su distribución de las varianzas durante la fase pretest. En segundo lugar, para estudiar la existencia de diferencias entre los cuatro grupos, se aplicó un segundo análisis de varianza (ANOVA), y posteriormente se realizó una prueba DMS para los análisis post-hoc comparando pares de grupos con respecto a las variables del Pro-Neuro. Se calculó el tamaño del efecto a través de dos estadísticos. Para estudiar toda la muestra se utilizó la eta cuadrado ( 12 ), mientras que la $d$ de Cohen se usó para hacer las comparaciones post-hoc entre los grupos en las variables medidas.

\section{Resultados}

$\mathrm{Al}$ analizar la fase pretest, los estadísticos de Levene y de Kolgomorov-Smirnov informaron que se cumplieron los supuestos de homocedasticidad y normalidad en las puntuaciones totales del Pro-Neuro antes de comenzar la intervención logopédica $\left(F_{(1,14)}=.224 ; \mathrm{p}<.50\right.$; $\left.D_{(8)}=.238, p<.50\right)$. El ANOVA mostró que existían diferencias significativas $\left(F_{(1,15)}=10.611 . ; p>.01\right)$ entre los sujetos que habían recibido el tratamiento farmacológico y los que no (Tabla 1).

El ANOVA de las variables del Pro-Neuro de la fase postest puso de manifiesto que existían diferencias significativas en las puntuaciones medias entre los grupos $\left(F_{(1,14)}=.224 ; p<.50\right)$. Los resultados en la puntuación total del Pro-Neuro indica que, por un lado, el tratamiento sigue protegiendo las capacidades cognitivas de los sujetos a los que se les administra $\left(F_{(1,15)}=248.06 ; p>\right.$ .027). Por otro lado, también se confirma que la intervención logopédica presenta beneficios significativos para los pacientes $\left(F_{(1,15)}=217.56 ; p>.036\right)$. Al realizar los análisis post-hoc de la fase postest se detectaron diferencias en 8 comparaciones de grupos $(p<.05)$, constatándose un menor nivel de cálculo mental, comprensión verbal, fluidez verbal, orientación temporal-espacial y puntuación total del Grupo Control cuando fue comparado con los otros tres grupos (Tabla 2).

\section{Discusión}

La presente investigación se articuló en torno a dos objetivos. En primer lugar, estudiar la efectividad de un programa piloto de intervención logopédica en pacientes diagnosticados de la EA en fase leve. En segundo lugar, comprobar si alguna de las áreas trabajadas mejoró o, al menos, quedó preservada tras finalizar el programa de

Tabla 1. Descriptivos y nivel de significación de las variables por grupo

\begin{tabular}{|c|c|c|c|c|c|c|c|c|c|c|c|}
\hline \multirow{3}{*}{$\begin{array}{c}\text { Grupo } \\
\text { Edad } \\
\text { Variable }\end{array}$} & \multicolumn{2}{|c|}{ Grupo 1 Control } & \multicolumn{2}{|c|}{$\begin{array}{l}\text { Grupo } 2 \text { Intervención } \\
\text { Logopédica }\end{array}$} & \multicolumn{2}{|c|}{$\begin{array}{l}\text { Grupo } 3 \text { Tratamiento } \\
\text { Farmacológico }\end{array}$} & \multicolumn{2}{|c|}{$\begin{array}{c}\text { Grupo } 4 \text { Tratamiento } \\
\text { Combinado }\end{array}$} & \multirow[b]{3}{*}{ 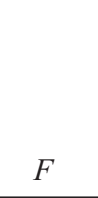 } & \multirow[b]{3}{*}{$\eta^{2}$} & \multirow[b]{3}{*}{$\alpha$} \\
\hline & \multicolumn{2}{|c|}{$75.8(3.2)$} & \multicolumn{2}{|c|}{$77(8.4)$} & \multicolumn{2}{|c|}{$76.8(9.5)$} & \multicolumn{2}{|c|}{$74(6.9)$} & & & \\
\hline & Pretest & Postest & Pretest & Postest & Pretest & Postest & Pretest & Pos & & & \\
\hline $\mathrm{AM}$ & $8(1.6)$ & $7.3(1.5)$ & 7 & $8(0.8)$ & $9.3(0.5)$ & 0 & 7 & 3) & 1.4 & 0.402 & 0.68 \\
\hline $\mathrm{CM}$ & $1.3(0.5)$ & $1(0)$ & $1.5(0.6)$ & $1.8(0.5)$ & $2(0)$ & $1.8(0.5)$ & $1.5(0.6)$ & $1.5(0.6)$ & 2.4 & 0.259 & 0.73 \\
\hline $\mathrm{CV}$ & $3(0.8)$ & $3(1.4)$ & $3.8(1)$ & $5(0)$ & $3.3(1.3)$ & $3.5(1.3)$ & $3.5(1.9)$ & $4.3(1.5)$ & 2.07 & 0.375 & 0.71 \\
\hline $\mathrm{D}$ & $9.5(0.6)$ & $9.3(1)$ & $8.3(0.5)$ & $9.3(0.5)$ & $9.5(0.6)$ & $9.3(0.5)$ & $10(0)$ & $9.8(0.5)$ & 0.0 & 0.341 & 0.73 \\
\hline E & $4.8(1.5)$ & $4.5(1.3)$ & $5(0.8)$ & $5.3(1)$ & $.5(1)$ & $5.5(1)$ & $5.3(1)$ & $5.8(0.5)$ & 1.22 & 0.233 & 0.73 \\
\hline FV & $0.5(1)$ & $0(0)$ & $1(0.8)$ & $2(0.8)$ & $2.8(1.3)$ & $2(1.6)$ & $2(0.8)$ & $2.5(1.3)$ & $3.93^{*}$ & 0.13 & 0.69 \\
\hline GV & $2.5(0.6)$ & $2.5(0.6)$ & $2.3(0.5)$ & $2.8(0.5)$ & $2.8(0.5)$ & $3(0)$ & $2.5(0.6)$ & $2.8(0.5)$ & 0.8 & 0.225 & 0.73 \\
\hline OTE & $3.5(1.3)$ & $2.8(1.7)$ & $3(2.2)$ & $3(2.7)$ & $3.8(2.2)$ & $3.5(1.7)$ & $5.3(1.5)$ & $6.3(1.5)$ & 2.69 & 0.496 & 0.7 \\
\hline $\mathrm{P}$ & $4.3(1.3)$ & $4.3(2.8)$ & $4.8(1)$ & $5.8(1)$ & $6.8(1.7)$ & $6.3(1)$ & $5.5(3)$ & $6(2.4)$ & 0.84 & 0.173 & 0.7 \\
\hline $\mathrm{R}$ & $0.8(1)$ & $0.5(1)$ & $1.5(1.3)$ & $1.5(1.3)$ & $2.5(0.6)$ & $1.3(1.5)$ & $1(1.4)$ & $2.3(1.5)$ & 1.16 & 0.167 & 0.7 \\
\hline Total & $38(4.5)$ & $35(6.5)$ & $38.3(5.2)$ & $44.3(6.6)$ & $48(5.7)$ & $44.8(7.2)$ & $44.3(4.8)$ & $50.3(4.3)$ & $4.12^{*}$ & 0.507 & 0.72 \\
\hline
\end{tabular}

Nota: Desviaciones típicas $(D T)$ de las medias entre paréntesis. $\mathrm{AM}=$ Atención y Memoria; $\mathrm{CM}=$ Cálculo Mental; $\mathrm{CV}=\mathrm{Comprensión}$ Verbal; D = Denominación; E = Escritura; FV = Fluidez Verbal; GV = Gnosia Visual; OTE = Orientación temporal-espacial; P = Praxias; $\mathrm{R}=$ Razonamiento; Total $=$ Puntuaciones totales del Pro-Neuro. ${ }^{*} p<.05$ 
Tabla 2. Diferencias entre cada par de grupos en todas las variables del Pro-Neuro

\begin{tabular}{|c|c|c|c|}
\hline $\begin{array}{c}\text { Nombre de la prueba } \\
\text { (variable) }\end{array}$ & Interpretación & $p$ & $d$ \\
\hline \multirow[t]{5}{*}{ CM (Cálculo Mental) } & $\mathrm{G} 1<\mathrm{G} 2$ & $0.024^{*}$ & 1.45 \\
\hline & $\mathrm{G} 1<\mathrm{G} 3$ & $0.024^{*}$ & 1.45 \\
\hline & $\mathrm{G} 1=\mathrm{G} 4$ & 0.134 & - \\
\hline & $\mathrm{G} 2=\mathrm{G} 3$ & 1 & - \\
\hline & $\mathrm{G} 2=\mathrm{G} 4$ & 0.537 & - \\
\hline \multirow[t]{5}{*}{ CV (Comprensión Verbal) } & $\mathrm{G} 1<\mathrm{G} 2$ & $0.03 *$ & 1.49 \\
\hline & $\mathrm{G} 1=\mathrm{G} 3$ & 0.62 & - \\
\hline & $\mathrm{G} 1=\mathrm{G} 4$ & 0.271 & - \\
\hline & $\mathrm{G} 2=\mathrm{G} 3$ & 0.059 & - \\
\hline & $\mathrm{G} 2=\mathrm{G} 4$ & 0.356 & - \\
\hline \multirow[t]{5}{*}{ FV (Fluidez Verbal) } & $\mathrm{G} 1<\mathrm{G} 2$ & $0.003^{*}$ & 1.42 \\
\hline & $\mathrm{G} 1<\mathrm{G} 3$ & $0.05^{*}$ & 1.42 \\
\hline & $\mathrm{G} 1<\mathrm{G} 4$ & $0.008^{*}$ & 1.78 \\
\hline & $\mathrm{G} 2=\mathrm{G} 3$ & 1 & - \\
\hline & $\mathrm{G} 2=\mathrm{G} 4$ & 0.537 & - \\
\hline \multirow{5}{*}{$\begin{array}{l}\text { OTE (Orientación tempo- } \\
\text { ral-espacial) }\end{array}$} & $\mathrm{G} 1=\mathrm{G} 2$ & 0.881 & - \\
\hline & $\mathrm{G} 1=\mathrm{G} 3$ & 0.56 & - \\
\hline & $\mathrm{G} 1<\mathrm{G} 4$ & $0.022 *$ & 1.54 \\
\hline & $\mathrm{G} 2=\mathrm{G} 3$ & 0.766 & - \\
\hline & $\mathrm{G} 2=\mathrm{G} 4$ & 0.081 & - \\
\hline Puntuaciones totales & $\mathrm{G} 1=\mathrm{G} 2$ & 0.091 & - \\
\hline \multirow[t]{4}{*}{ Pro-Neuro } & $\mathrm{G} 1=\mathrm{G} 3$ & 0.091 & - \\
\hline & $\mathrm{G} 1<\mathrm{G} 4$ & $0.008^{*}$ & 1.92 \\
\hline & $\mathrm{G} 2=\mathrm{G} 3$ & 0.922 & - \\
\hline & $\mathrm{G} 2=\mathrm{G} 4$ & 0.176 & - \\
\hline
\end{tabular}

Nota: Estadístico para la prueba de contraste DMS (IC 95\%). $d=$ tamaño del efecto ( $d$ de Cohen).

intervención. Los resultados de la investigación dieron por cumplidos los objetivos y ofrecieron resultados destacables. Se encontró que en la fase pretest el tratamiento farmacológico con ICE fue eficaz frenando el deterioro de los síntomas del Alzheimer, replicando los resultados encontrados en la literatura científica (Sun et al., 2012; Kumar et al., 2015; Boccardi et al., 2016). En segundo lugar, se observó que la aplicación de la intervención logopédica fue efectiva. Los grupos que recibieron el entrenamiento durante las 18 sesiones no empeoraron en ninguna de las áreas intervenidas, e incluso presentaron mejorías en el postest en las puntuaciones totales del Pro-Neuro (Figura 1) y en fluidez verbal. Además, el grupo combinado mostró los mejores resultados al beneficiarse de dos procedimientos dedicados en exclusiva a la preservación de sus capacidades lingüísticas y cognitivas. Estos resultados dan por confirmados los objetivos de la presente investigación y cumplen la hipótesis de trabajo.

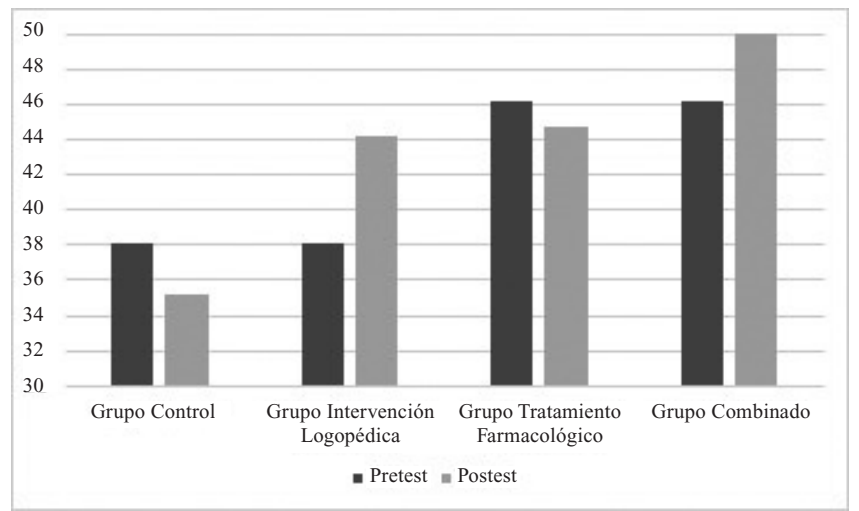

Figura 1. Puntuaciones totales de la Escala Pro-Neuro de Evaluación Cognitiva-Lingüística.

Nota: La escala Pro-Neuro tiene como máximo de puntuación un 100.

Con respecto a los datos, debemos hacer hincapié en los resultados de fluidez verbal. Esta área fue la más beneficiada, presentando mejorías en los grupos de intervención logopédica y en el grupo combinado, y decayendo en el grupo control. Esto, si bien es llamativo, no es sorprendente. En 2009, Iacono y cols., llevaron a cabo un estudio en la observaron el cerebro de 28 religiosas católicas tras su muerte y compararon los escritos que se conservaban de ellas cuando ingresaron en el convento, normalmente a la edad de veinte años. Dividieron la muestra en función a si tenían o no indicadores de la EA, para después medir la fluidez verbal de los textos de su juventud. Los resultados mostraron que las mujeres que desarrollaron indicadores de la EA tenían una puntuación en fluidez un 20\% mas bajo. Extrapolado a nuestros resultados, el éxito de la intervención nos indica que la fluidez puede ser tomada tanto como un indicador de protección ante la EA como un predictor temprano de su desarrollo cuando sea baja. Además, se constata que, al contrario que la mayoría de las áreas logopédico-cognitivas que únicamente frenan su deterioro, la fluidez se ve altamente beneficiada cuando es intervenida directamente.

A partir de los resultados obtenidos podemos extraer varias conclusiones. En primer lugar, aunque el tratamiento colinérgico presenta algunas contraindicaciones (Hernández-Arroyo y Díaz-Madero, 2016), se ha comprobado que es efectivo para detener el avance de la EA en la mayoría de las áreas cognitivas estudiadas. En segundo lugar, aunque este tratamiento sea beneficioso, solo retrasa el inevitable deterioro cognitivo que produce la EA. La administración en paralelo de un tratamiento logopédico es una manera eficaz de frenar el deterioro en sus habilidades cognitivas, retrasando el paso de la en- 
fermedad a su fase moderada. Sin embargo, esto no es ninguna solución permanente, y es necesario mucho más trabajo de investigación e intervención para luchar contra los devastadores efectos de esta enfermedad.

Otra conclusión extraída a partir de los datos pone el foco en los resultados del grupo control y en la necesidad de aplicar algún tipo de intervención sistemática a los enfermos de la EA. Estos sujetos no solo tuvieron puntuaciones más bajas desde el inicio, sino que empeoraron tras los tres meses que duró la investigación. Debemos reflexionar sobre las consecuencias a nivel cognitivo que tiene el no aplicar ninguna terapia paliativa de la EA en fase leve. Un gran número de investigaciones clínicas se están orientando a encontrar estrategias de prevención temprana de esta enfermedad (Crous-Bou, Minguillón, Gramunt, y Molinuevo, 2017), puesto que cuanto antes se ataje el deterioro de la enfermedad, más tiempo se mantendrá su calidad de vida. Esto se está viendo favorecido por importantes avances en la búsqueda de predictores tempranos de la EA (Laske et al., 2015), tales como biomarcadores en el fluido cerebro espinal (Blennow et al., 2018), marcadores en sangre (O'Bryant et al., 2010), historial de demencias (Barnes et al., 2012), técnicas de neuroimagen (Ortiz, Munilla, Álvarez-Illán, Górriz, y Ramírez, 2015) o incluso historial de infecciones bucales (Dominy et al., 2019). Todo esto nos impulsa a afirmar que es necesario desarrollar herramientas como la presente intervención logopédica, que se pueden implementar de manera económica, rápidamente y sin efectos secundarios desde el mismo momento en el que se sospecha la presencia de la enfermedad. Mientras la investigación en predictores avanza, las intervenciones cognitivas, logopédicas o farmacológicas son, actualmente, una reacción indispensable pero tardía ante el progreso de una enfermedad devastadora.

Además de los resultados este estudio piloto, es necesario dar visibilidad al papel del logopeda en las intervenciones clínicas en personas mayores y con demencias. Formados específicamente con conocimientos teórico-prácticos especializados, los logopedas deberían ser parte integrante de cualquier equipo de atención primaria que trabaje con esta población. Sin embargo, esto no sucede. Según una reciente encuesta del Colegio Oficial de Logopedas de la Comunitat Valenciana (COLCV, 2018), sólo el 10\% de los logopedas trabaja en el ámbito sociosanitario relacionado con mayores. Esto es un porcentaje muy limitado para unos profesionales de la salud con una capacitación específica en trastornos de la comunicación, y que puede aportar mucho a la práctica clínica. Esta investigación muestra que el estudio y la intervención de las habilidades lingüísticas en pacientes con demencias neurodegenerativas es un campo que ne- cesita el mayor número de profesionales, aproximaciones prácticas y perspectivas teóricas posibles.

Para finalizar, nos gustaría concluir con algunas limitaciones de este estudio piloto que deberían de tenerse en cuenta a la hora de una posible replicación. La potencia estadística de las comparaciones entre grupos no es elevada, debido al reducido número de sujetos. Aunque este tipo de investigaciones depende, en gran medida, de la posibilidad de encontrar una muestra tan específica, sería muy deseable repetir este estudio con un número muestral más elevado. En dicha replicación sería muy informativo el prestar atención a las tareas de fluidez verbal y comprobar cómo y en qué medida esta área sigue mejorando en los pacientes y si pudiera ser utilizado como un predictor de la EA. En todo caso, una de las ventajas de este protocolo logopédico es su brevedad, puesto que los enfermos de la EA se cansan con facilidad y no toleran intervenciones largas. Si se comprobase que la fluidez puede mejorar con intervenciones más breve, esto sería de interés puesto que se podría ajustar el tiempo de las sesiones para trabajar otras áreas más deterioradas, con la consiguiente utilidad clínica.

\section{Conflictos de intereses}

Los autores declaran que no existen conflictos de intereses.

\section{Referencias}

Adrián, J. A., Hermoso, P., Buiza, J. J., Rodríguez-Parra, M. J., y González, M. (2008). Estudio piloto de la validez, fiabilidad y valores de referencia normativos de la escala PRO-NEURO en adultos mayores sin alteraciones cognitivas. Neurología, 23, 275-287.

Aguirre, E., Spector, A., Hoe, J., Russell, I. T., Knapp, M., Woods, R. T., \& Orrell, M., (2010). Maintenance Cognitive Stimulation Therapy (CST) for dementia: A single-blind, multi-centre, randomized controlled trial of Maintenance CST vs. CST for dementia. Trials, 46, 11-46.

Aranda, L., Gordillo-Villatoro, M., Enamorado, J. M., y López-Zamora, M. (2017). Estudio del lenguaje en las diferentes fases de la enfermedad de Alzheimer a través del Test de Boston. Revista de Logopedia, Foniatría y Audiología, 37, 111-120.

Barnes, D. E., Yaffe, K., Byers, A. L., McCormick, M., Schaefer, C., \& Whitmer R. A. (2012). Midlife vs late-life depressive symptoms and risk of dementia: differential effects for Alzheimer disease and vascular dementia. Archives of General Psychiatry, 69, 493-498.

Blennow, K., Dubois, B., Fagan, A. M., Lewczuk, P., Leon, M. J., \& Hampel, H. (2015). Clinical utility of cerebrospinal fluid biomarkers in the diagnosis of early Alzheimer's disease. Alzheimer's \& dementia: the journal of the Alzheimer's Association, 11, 58-69. 
Boccardi, V., Baroni, M., Smirne, N., Clodomiro, A., Ercolani, S., Longo, A., ... \& Mecocci, P. (2017). Short-Term Response is not Predictive of Long-Term Response to Acetylcholinesterase Inhibitors in Old Age Subjects with Alzheimer's Disease: A «Real World» Study. Journal of Alzheimer Disease, 56, 239248.

Burge, E., Kuhne, N., Berchtold, A., Maupetit, C., \& von Gunten, A. (2011). Impact of physical activity on activity of daily living in moderate to severe dementia: a critical review. European Review of Aging and Physical Activity, 9(1), 27-39.

Burns, A. \& Iliffe, S. (2009). Alzheimer's disease- clinical review. BMJ 338, 467-471.

Colegio Oficial de Logopedas de la Comunitat Valenciana. Encuesta sobre la situación actual de la profesión de logopedia 2016 (17 de mayo de 2018). Recuperado de http://www.colegiologopedas-cv.org/

Crous-Bou, M., Minguillón, C., Gramunt, N., \& Molinuevo, J. L. (2017). Alzheimer's disease prevention: from risk factors to early intervention. Alzheimer's Research \& Therapy, 9, 71.

Cullell, N., Bruna, O., y Puyuelo, M. (2006). Intervención neuropsicológica y del lenguaje en la enfermedad de Alzheimer. Descripción de un caso clínico. Revista de Logopedia, Foniatría y Audiología, 26(4), 231-238.

Dominy, S. S., Lynch, C., Ermini, F., Benedyk, M., Marczyk, A., Konradi, A., ... \& Potempa, J. (2019). Porphyromonas gingivalis in Alzheimer's disease brains: Evidence for disease causation and treatment with small-molecule inhibitors. Science Advances, 5, 123-145.

Fernández-Calvo, B., Contador, I., Serna, A., Menendez de Lucena, V., y Ramos, F. (2010) Efecto del formato de intervención individual o grupal en la estimulación cognitiva de pacientes con enfermedad de Alzheimer. Revista de Psicopatología y Psicología, 15, 115-123.

Folstein, M. F., Folstein, S. E., McHugh, P. R., \& Fanjiang, G. (2000). Mini-Mental State Examination: MMSE user's guide. Odessa, Fla. Psychology Assessment Resources.

Frederiksen, K. S., Gjerum, L., Waldemar, G., \& Hasselbalch, S. G. (2017). Effects of Physical Exercise on Alzheimer's Disease Biomarkers: A Systematic Review of Intervention Studies. Journal of Alzheimer's Disease, 61, 359-372.

Gallucci, M., Spagnolo, P., Aricó, M., \& Grossi, E. (2016). Predictors of response to cholinesterase inhibitors treatment of Alzheimer's disease: Data mining from the TREDEM registry. Journal of Alzheimer's Disease, 50, 969-979.

García-Alberca, J. M. (2015). Las terapias de intervención cognitiva en el tratamiento de los trastornos de conducta en la enfermedad de Alzheimer. Evidencias sobre su eficacia y correlaciones neurobiológicas. Neurología, 30(1), 8-15.

García-Casares, N., Moreno-Leiva, R. M., y García-Arnés, J. A. (2017). Efecto de la musicoterapia como terapia no farmacológica en la enfermedad de Alzheimer. Revisión sistemática. Revista de Neurología, 65, 529-538.

Hernández-Arroyo, M. J., y Díaz-Madero, A. (2016). Valoración del riesgo/beneficio en el tratamiento de la enfermedad de Alzheimer. A propósito de una interacción. Revista Española de Geriatría y Gerontología, 4, 187-248.

Iacono, D., Markesbery, W. R., Gross, M., Pletnikova, O., Rudow, G., Zandi, P., \& Troncoso, J. C. (2009). The Nun Study. Neurology, 73, 665-673.
Klimova, B., Maresova, P., Valis, M., Hort, J., \& Kuca, K. (2015). Alzheimer's disease and language impairments: Social intervention and medical treatment. Clinical Interventions in Aging, 10, 1401-1408.

Kumar, A., Singh, A., \& Ekavali. (2015). A review on Alzheimer's Disease pathophysiology and its management: An update. Pharmacological Reports, 67, 105-203.

Laske, C., Sohrabi, H. R., Frost, S. M., López-de-Ipiña, K., Garrard, P., Buscema, M., ... \& O'Bryant, S. E. (2015). Innovative diagnostic tools for early detection of Alzheimer's disease. Alzheimers \& Dementia, 11, 561-578.

Livingston, G., Sommerlad, A., Orgeta, V., Costafreda, S. G, Huntley, J., Ames, D., ... \& Mukadam, N. (2017). Dementia prevention, intervention, and care. Lancet, 390, 2673-734.

Llanero, M., Ruiz, J. M., Medrano, P., y Fernández, C. (2011). Tratamiento de la enfermedad de Alzheimer. Medicine, 10, 5138-5144.

Lobo, A., Saz, P., Marcos, G., Día, J. L., De la Cámara, C., ... y Ventura, T. (1999). Revalidación y normalización del miniexamen cognoscitivo (primera versión en castellano del Minimental State Examination) en la población general geriátrica. Medicina Clínica, 112, 767-774.

López Locanto, O. (2015). Tratamiento farmacológico de la enfermedad de Alzheimer y otras demencias. Archivos de Medicina Interna, 37, 61-67.

Mielke, M. M., Vemuri, P., \& Rocca, W. A. (2014). Clinical epidemiology of Alzheimer's disease: assessing sex and gender differences. Clinical Epidemiology, 6, 37-48.

Muñoz Lasa, S., Máximo Bocanegra, N., Valero Alcaide, R., Atín Arratibel M. A., Varela Donoso, E., y Ferriero, G. (2015). Animal assisted interventions in neurorehabilitation: a review of the most recent literature. Neurología, 30, 1-7.

Niu, H. X., Álvarez-Álvarez, I., Guillén-Grima, F., y Aguinaga-Ontoso, I. (2017). Prevalencia e incidencia de la enfermedad de Alzheimer en Europa: metaanálisis. Neurología, 32, 523-532.

Novoa, A. M., Juárez, O, y Nebot, M. (2008). Efectividad de las intervenciones cognitivas en la prevención del deterioro de la memoria en las personas mayores sanas. Gaceta Sanitaria, 22, 474-482.

O’Bryant S. E., Xiao G., Barber R., Reisch J., Doody R., Fairchild T., .. \& Diaz-Arrastia, R. (2010). A serum protein-based algorithm for the detection of Alzheimer disease. Archives of Neurology, 67, 1077-1081.

Olazarán, J., Reisberg, B., Clare, L., Cruz, I., Peña-Casanova, J., ... y Muñiz, R. (2010). Eficacia de las terapias no farmacológicas en la enfermedad de Alzheimer: una revisión sistemática. Dementia and Geriatric Cognitive Disorders, 30, 161-178.

Ortiz, A., Munilla, J., Álvarez-Illán, I., Górriz, J. M., y Ramírez, J. (2015). Exploratory graphical models of functional and structural connectivity patterns for Alzheimer's Disease diagnosis. Frontiers in Computational Neuroscience, 9, 132.

Peña-Casanova, J. (1999). Enfermedad de Alzheimer. Del diagnóstico a la terapia: conceptos y hechos. Barcelona: Fundación «la Caixa».

Real Pérez, M., Robles Rodríguez, C., y Ponce González, J. (2017). Revisión narrativa y desarrollo de un programa de intervención para la disminución de los efectos del Alzheimer a través de la práctica del Surf en Personas Mayores. Retos, 32, 106-110. 
Ruiz-Sánchez de León, J. M. (2012). Estimulación cognitiva en el envejecimiento sano, el deterioro cognitivo leve y las demencias: estrategias de intervención y consideraciones teóricas para la práctica clínica. Revista de Logopedia, Foniatría y Audiología, 32, 57-66.

Sun, X., Jin, L., \& Ling, P. (2012). Review of drugs for Alzheimer'sdisease. Drug Discoveries \& Therapeutics, 6, 285290.

Tárraga, L. (2001). Tratamientos de psicoestimulación. En: Fernández-Ballesteros, R, Nicolás J, eds. Libro blanco sobre la Enfermedad de Alzheimer (pp. 305-323). Madrid: Obra Social de Caja Madrid.

Tárraga, L., y Boada, M. (1999). Volver a empezar. Ejercicios prácticos de estimulación cognitiva para enfermos de Alzheimer. Barcelona: Glosa.
United Nations, Department of Economic and Social Affairs, Population Division (2017). World Population Ageing 2017 (ST/ ESA/SER.A/408). (13 de marzo de 2019). Recuperado de http://www.un.org/en/development/desa/population/publications/pdf/ageing/WPA2017_Report.pdf

Valenzuela, M. J. (2008). Brain reserve and the prevention of dementia. Current Opinion in Psychiatry 21, 296-302.

Van der Kleij, L. A., Petersen, E. T., Siebner, H. R., Hendrikse, J., Frederiksen, K. S., ... \& Garde, E. (2018). The effect of physical exercise on cerebral blood flow in Alzheimer's disease. NeuroImage: Clinical, 20, 650-654.

Zamarrón, M. D., Tárraga, L., y Fernández-Ballesteros, R. (2008). Plasticidad cognitiva en personas con la enfermedad de Alzheimer que reciben programas de estimulación cognitiva. Psicothema, 20, 432-437. 